\title{
Seven-Day Bismuth-based Quadruple Therapy as an Initial Treatment for Helicobacter pylori Infection in a High Metronidazole Resistant Area
}

\author{
Ratha-korn Vilaichone ${ }^{1,5 *}$, Hatainuch Prapitpaiboon ${ }^{1}$, Pornpen Gamnarai ${ }^{2,5}$, \\ Juraiwan Namtanee ${ }^{1,5}$, Arti Wongcha-um ${ }^{3}$, Supakarn Chaithongrat ${ }^{4}$, Varocha \\ Mahachai ${ }^{4,5}$
}

\begin{abstract}
Background: The prevalence of metronidazole-resistant $\mathrm{H}$. pylori is almost $50 \%$ in Thailand which severely limits the use of this drug for eradication therapy. The aims of this study were to evaluate the efficacy and safety profiles of 7-day bismuth-based quadruple therapy including metronidazole as an initial treatment for $\boldsymbol{H}$. pylori infection in a high metronidazole resistance area. Materials and Methods: This study was performed at Thammasat University Hospital and King Chulalongkorn Memorial Hospital during January 2009 to October 2010. Patients with non-ulcer dyspepsia (NUD) with active $H$. pylori infection were assigned to receive seven days of quadruple therapy (pantoprazole $40 \mathrm{mg}$ bid, bismuth subsalicylate $1,048 \mathrm{mg}$ bid, amoxicillin $1 \mathrm{gm}$ bid and metronidazole $400 \mathrm{mg}$ tid). $H$. pylori infection was defined as positive $H$. pylori culture or two positive tests (rapid urease test and histology). Antibiotic susceptibility test for metronidazole by Epsilometer test (E-test) was performed in all positive cultures. At least four weeks after treatment, ${ }^{13} \mathrm{C}$ urea breath test $\left({ }^{13} \mathrm{C}\right.$-UBT $)$ was performed to confirm $H$. pylori eradication. Results: A total of 114 patients were enrolled in this study, 50 males and 64 females with a mean age of 49.8 years. All 114 patients had a diagnosis of NUD. Overall eradication as confirmed by negative ${ }^{13} \mathrm{C}$-UBT was achieved in 94 out of 114 patients $(82.5 \%) .44$ patients had positive cultures and success for E-test. In vitro metronidazole resistance was observed in $22 / 44(50 \%)$ patients. Eradication rate in patients with metronidazole resistant strains was $16 / 22(72.7 \%)$ and $20 / 22(90.1 \%)$ with metronidazole sensitive strains $(72.7 \%$ vs $90.1 \%$, p-value $=0.12 ; \mathrm{OR}=3.75$ [95\% CI=0.6-31.5]). Minor adverse reactions included nausea, bitter taste, diarrhea and black stools but none of the patients dropped out from the study. Conclusions: Initial treatment with 7-day bismuth-based quadruple therapy including metronidazole, amoxycillin and pantoprazole is highly effective and well tolerated for metronidazole-sensitive $H$. pylori infections. However, the efficacy markedly decline with metronidazole resistance. Longer duration of this regimen might be required to improve the eradication rate and larger multi-center studies are needed to confirm this hypothesis.
\end{abstract}

Keywords: Bismuth - quadruple therapy - Helicobacter pylori eradication - Thailand

Asian Pac J Cancer Prev, 16 (14), 6089-6092

\section{Introduction}

Helicobacter pylori is the common chronic infection leading to gastritis, peptic ulcer disease, gastric cancer and gastric lymphoma. (Vilaichone et al., 2001; Vilaichone, et al 2006). Effective eradication regimens are required to reduce the consequences of $H$. pylori infection. Standard triple therapy is commonly used regimen recommended by many guidelines. However, the efficacy of triple therapy has recently decline worldwide to $70-75 \%$ which is unacceptable (Vilaichone et al., 2006; Vilaichone et al, 2011). Improvement of eradication regimens depends on local data of antibiotic resistant pattern in each particular area. Metronidazole resistance has been detected approximately 40-50\% in Thailand (Vilaichone et al, 2013). Metronidazole resistance, however, has lesser impact on eradication rate compared to clarithromycin resistance. Bismuth has anti-H. pylori property that could suppress the bacterial load and was wildly used in quadruple therapy (Vilaichone et al., 2006). It has been suggested that bismuth-based quadruple therapy is either as effective as or more effective than standard triple therapy and might be used as first-line treatment (Ferreira and Moss et al., 2014).

${ }^{1}$ Gastroenterology Unit, Department of Medicine, ${ }^{2}$ Department of Biochemistry, Thammasat University Hospital, Pathumthani, ${ }^{4}$ Division of Gastroenterology, Department of Medicine, Chulalongkorn University Hospital, ${ }^{5}$ National Gastric Cancer and Helicobacter pylori Research Center, Bangkok, Thailand, ${ }^{3}$ School of Medicine, University of Southampton, United Kingdom *For correspondence: Vilaichone@hotmail.co.th 
Newer regimens are required in Thailand for effective H. pylori eradication. This study was aimed to evaluate the efficacy and safety of bismuth containing quadruple regimen as first line therapy for $H$. pylori eradication in Thailand. We also compared the eradication rates of this quadruple therapy between metronidazole resistant and sensitive groups.

\section{Materials and Methods}

Dyspeptic patients who had performed upper GI endoscopy and found to have H.pylori infection confirmed by positive $H$. pylori culture; or two positive tests (rapid urease test and histology) were recruited into this study at Thammasat University Hospital and King Chulalongkorn Memorial Hospital during January 2009 to October 2010. Patients must be over 18 years of age and had never received $H$. pylori treatment or received proton pump inhibitor, $\mathrm{H} 2$ receptor antagonists, and any antibiotics in the past 1 month. More exclusion criteria included patients with a history of prior gastric surgery, pregnancy, major systemic diseases such as cardiovascular disease, chronic renal and liver diseases or history of allergy to any one of medication in the regimens. Written Informed consents were done before participating in this study. Diagnosis was made according to endoscopic findings. Patients with normal endoscopy and those with gastritis were diagnosed to have non-ulcer dyspepsia (NUD). During endoscopy, three biopsy samples from gastric antrum were collected; one for rapid urease test, one for histology and another biopsy for $H$. pylori culture and antibiotic susceptibility test.

\section{H. pylori culture and antibiotic susceptibility test}

H. pylori isolates were obtained by inoculating the specimens on nonselective media (Columbia agar supplemented with $7 \%$ sheep blood) under a microaerophilic atmosphere for at least 7 days. The colonies were identified by gram straining, oxidase, catalase and urease production. Susceptibility for metronidazole was tested by the Epsilometer test (E-test). The minimum inhibitory concentration (MIC) was defined as the lowest concentration of antimicrobial agent which completely inhibited any visible growth. Metronidazole resistance was defined as MIC above $8 \mu \mathrm{g} / \mathrm{ml}$.

\section{Treatment regimen}

Only those patients with $H$. pylori infection received seven days of quadruple therapy consisting of pantoprazole $40 \mathrm{mg}$ bid, bismuth subsalicylate $1,048 \mathrm{mg}$ bid, amoxicillin $1 \mathrm{gm}$ bid and metronidazole $400 \mathrm{mg}$ tid. Patients did not receive further PPI therapy.

\section{Post-treatment follow up}

Patients returned for follow-up visit at the end of the eradication therapy for assessment of compliance and side effects. Pill count was conducted and drug consumption over $90 \%$ was defined as good compliance. Side effects were assessed by personal interview using opened ended questions. New symptoms and exacerbation of pre-existing symptoms during the treatment period were considered to be therapy-related side effects. ${ }^{13} \mathrm{C}$ urea breath test (UBT) was performed to assess $H$. pylori eradication at least 4 weeks after completion of the treatment.

\section{Statistical analysis}

We expected the eradication rate of 7-day bismuthbased quadruple therapy as an empiric therapy to be $\geq 90 \%$. Treatment success was identified as a cure rate of $\geq 95 \%$ (i.e. grade A) as described in previous studies (Graham et al., 2007), and failure as a cure rate of $<90 \%$ per protocol. The association between eradication rate of patients with metronidazole resistance and sensitive was evaluated by using chi-squared. Association between categorical variables were determined using odds ratio and $95 \%$ confidence interval $(95 \% \mathrm{CI})$. The $\mathrm{p}$ values $<0.05$ were considered to be statistically significant. The data analysis was performed by using SPSS version 19 (SPSS Inc., Chicago, IL, USA). The study was conducted according to the good clinical practice guidelines, as well as the Declaration of Helsinki and was approved by our local ethics committee.

\section{Results}

A total of 114 patients were enrolled in this study. There were 50 male and 64 female patients with the mean age of 49.8 years. All 114 patients had diagnosis of NUD. Overall eradication rate as confirmed by negative ${ }^{13} \mathrm{C}$-UBT was achieved in 94 out of 114 patients $(82.5 \%)$. 44 patients had positive cultures and success for E-test. In vitro metronidazole resistance determined by E-test was identified in 22/44 (50\%) of these patients. Eradication rate of patients with metronidazole resistant strain was $16 / 22(72.7 \%)$ and $20 / 22(90.1 \%)$ with metronidazole

\section{Table 1. Adverse Events in All Patients}

\begin{tabular}{lc}
\hline Adverse events & $\begin{array}{c}\text { 7-day bismuth-based quadruple therapy } \\
(\mathrm{n}=114)\end{array}$ \\
\hline Bitter taste & $38(33.3 \%)$ \\
Nausea/Vomiting & $14(12.3 \%)$ \\
Diarrhea & $3(2.6 \%)$ \\
Black stool & $102(89.5 \%)$ \\
\hline
\end{tabular}

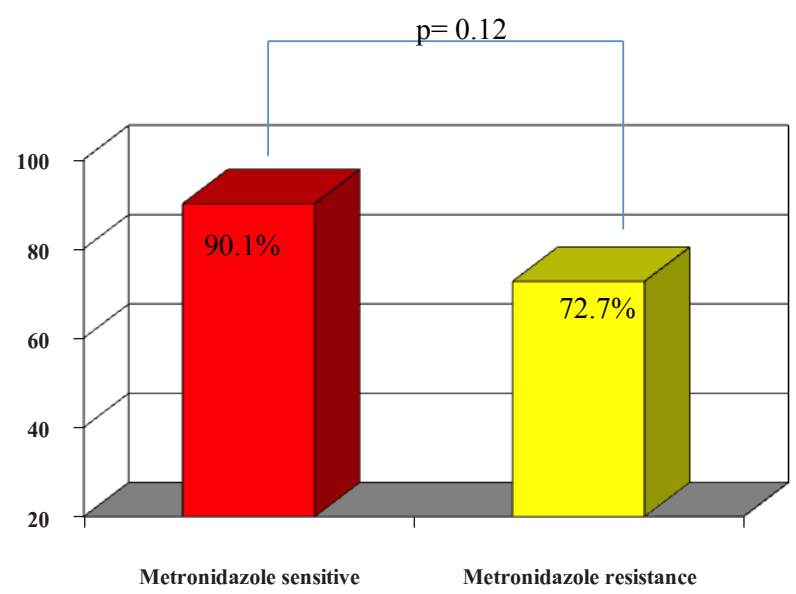

Figure 1. H. pylori Eradication Rate Of 7-Day BismuthBased Quadruple Therapy in Metronizdazole Resistant and Metronidazole Sensitive Strains 
sensitive strains $(72.7 \%$ vs $90.1 \%$, p-value $=0.12 ; \mathrm{OR}=3.75$ [95\% CI=0.6-31.5]). Minor adverse reactions were commonly occurred included nausea, bitter taste, diarrhea and black stools as detail in Table 1. None of the patients dropped out from the study. The side effects disappeared after cessation of the medications.

\section{Discussion}

Many epidemiological and experimental data demonstrated a significant role between $H$. pylori and pathogenesis of gastric cancer. It was estimated approximately 700,000 new cases of gastric cancer annually and gastric cancer was document to be the first GI cancer related death. (Rauws and Tytgat, 1990; Demirel et al., 2013; Basiri et al., 2014; Srinarong et al., 2014; Vilaichone et al., 2014; Naghavi et al., 2015). The eradication of $H$. pylori by the high efficacy regimen should be the appropriate tool to reduce and prevent the development of this particular cancer. Standard triple therapy is no longer recommended as a first regimen in several countries (Chey and Wong, 2007; Mahachai et al., 2011). Bismuth based quadruple therapy might be an alternative first line regimen even in metronidazole resistance era. Metronidazole resistance has little impact on eradication rate of quadruple therapy and can be used effectively in the area with high metronidazole resistance (Vilaichone et al., 2006).

Bismuth has long become available in Thailand with an inexpensive price and acceptable side effects such as nausea, vomiting, numbness and metallic taste. Another possible adverse reaction of bismuth is black stools which should be warned to patients and should not confuse with melena from upper GI bleeding. Bismuth has antibacterial activity and prevents bacterial colonization to gastric epithelium and has no prior resistance report to $H$.pylori (Vilaichone et al., 2006,). This study demonstrated high eradication rate $(>90 \%)$ of a 7 -day bismuth based quadruple regimen in metronidazole sensitive strain. However, the efficacy of this regimen was reduced to $72.7 \%$ in metronidazole resistant group. A longer duration of treatment (eg. 14 days) might give a higher eradication rate but perhaps more side effects can be occurred. In Thailand, 10-day course of sequential therapy and 10-day concomitant therapy provided high $H$. pylori eradication rate $(>95 \%)$ (Mahachai et al., 2011; Kongchayanun et al., 2012). Thus, sequential and concomitant therapies might be reliable first line for $H$. pylori eradication in our country. Recently, another study from Thailand which used 14-day levofloxacin-dexlansoprazole quadruple therapy demonstrated high eradication rate of $H$. pylori infection regardless of CYP2C19 genotype, clarithromycin or dual clarithromycin and metronidazole resistant strains (Prapitpaiboon et al., 2015). However, this new quadruple therapy should consider testing in other clinical trials before granting as a first line $H$. pylori eradication in our country.

A seven day bismuth-based quadruple therapy is highly effective eradication regimen that is well tolerated in metronidazole-sensitive H.pylori. However, the efficacy was markedly decline in metronidazole resistance. Longer duration of this regimen might be required to improve the eradication rate and larger multi-center studies are needed to confirm this hypothesis.

\section{Acknowledgements}

This work was partially supported by the National Research University Project of Thailand Office of Higher Education Commission and the Excellence Center GI Endoscopy Research Fund at King Chulalongkorn Memorial Hospital.

\section{References}

Basiri Z, Safaralizadeh R, Bonyadi MJ, et al (2014). Helicobacter pylori vacA d1 genotype predicts risk of gastric adenocarcinoma and peptic ulcers in northwestern Iran. Asian Pac J Cancer Prev, 15, 1575-9.

Chey WD, Wong BC (2007). American College of Gastroenterology guideline on the management of Helicobacter pylori infection. Am J Gastroenterol, 102, 1808-25.

Demirel BB, Akkas BE, Vural GU (2013). Clinical factors related with Helicobacter pylori infection-is there an association with gastric cancer history in first-degree family members? Asian Pac J Cancer Prev, 14, 1797-802.

Ferreira J, Moss SF (2014). Current Paradigm and Future Directions for Treatment of Helicobacter pylori Infection. Curr Treat Options Gastroenterol, 12, 373-84.

Graham DY, Lu H, Yamaoka Y (2007). A report card to grade Helicobacter pylori therapy. Helicobacter, 12, 275-8.

Kongchayanun C,Vilaichone RK, Pornthisarn B, et al (2012). Pilot studies to identify the optimum duration of concomitant Helicobacter pylori eradication therapy in Thailand. Helicobacter, 17, 282-5.

Mahachai V, Sirimontaporn N, Tumwasorn S, Thong-Ngam D, Vilaichone RK (2011). Sequential therapy in clarithromycinsensitive and -resistant Helicobacter pylori based on polymerase chain reaction molecular test. J Gastroenterol Hepatol, 26, 825-8.

Naghavi M, Wang H, Lozano R, et al (2015). Global, regional, and national age-sex specific all-cause and cause-specific mortality for 240 causes of death, 1990-2013: a systematic analysis for the global burden of disease study 2013. Lancet, 385, 117-71.

Prapitpaiboon H, Mahachai V, Vilaichone RK (2015). High efficacy of levofloxacin-dexlansoprazole-based quadruple therapy as a first line treatment for Helicobacter pylori eradication in Thailand. Asian Pac J Cancer Prev, 16, 4353-6.

Rauws EA, Tytgat GN (1990). Cure of duodenal ulcer associated with eradication of Helicobacter pylori. Lancet, 335, 1233-5.

Srinarong C, Siramolpiwat S, Wongcha-um A, Mahachai V, Vilaichone RK (2014). Improved eradication rate of standard triple therapy by adding bismuth and probiotic supplement for Helicobacter pylori treatment in Thailand. Asian Pac J Cancer Prev, 15, 9909-13.

Vilaichone RK, Gumnarai P, Ratanachu-Ek T, Mahachai V (2013). Nationwide survey of Helicobacter pylori antibiotic resistance in Thailand. Diagn Microbiol Infect Dis, 77, 346-9.

Vilaichone RK, Mahachai V (2001). Current management of Helicobacter pylori infection. J Med Assoc Thai, 84, 32-38.

Vilaichone RK, Mahachai V, Graham DY (2006). Helicobacter pylori diagnosis and management. Gastroenterol Clin North Am, 35, 229-47. 


\section{Ratha-korn Vilaichone et al}

Vilaichone RK, Mahacahai VM, Tumwasorn S, et al (2011). CagA genotype and Metronidazole resistant strain of Helicobacter pylori in Functional dyspepsia in Thailand. $J$ Gastroenterol Hepatol, 26, 46-8.

Vilaichone RK, Panarat W, Aekpongpaisit S, Mahachai V (2014). Clinical characteristics and survival of gastric cancer in Thailand. Asian Pac J Cancer Prev, 15, 9005-8. 